\title{
A Cognitive Radio based Solution to Coexistence of FH and OFDM Signals Implemented on USRP N210 Platform
}

\author{
Miloš Janjić and Miljko Erić
}

\begin{abstract}
A new concept development and practical implementation of an OFDM based secondary cognitive link are presented in this paper. Coexistence of a secondary user employing Orthogonal Frequency Division Multiplexing (OFDM) and a primary user employing Frequency Hopping $(\mathrm{FH})$ is achieved. Secondary and primary links are realized using Universal Software Radio Peripheral (USRP) N210 platforms. Cognitive features of spectrum sensing and changing transmission parameters are implemented. Some experimental results are presented.
\end{abstract}

Keywords - Cognitive radio, FH, OFDM, secondary link, spectrum sensing, USRP platform.

\section{INTRODUCTION}

$\mathrm{T}_{\mathrm{t}}$ HE growth of wireless communication has created new technologies, which provide services with a high bandwidth and lead to an increase in the usage of radio spectrum. It was reported that some parts of the allocated spectrum at some locations were unused most of the time [1]. The scarcity of spectrum and need for efficient usage prompted research aimed at finding some other, more flexible, way of spectrum use, which has led to the development of a cognitive radio concept [2]. The basic idea is to make the spectrum access more flexible, i.e., to allow unlicensed users to use the licensed spectrum. In the cognitive radio (CR) terminology, licensed users are called primary users, while unlicensed users are called secondary or cognitive users. A secondary link must be able to dynamically change transmission parameters in order not to make interference towards primary users.

The most important task in CR is spectrum sensing, that includes the process of spectrum holes detection and analysis of signals active in some part of the spectrum. An excellent survey of spectrum sensing methods for CR with challenges associated is given in [3].

Paper received April 27, 2015; revised October 28, 2106; accepted Feb 2, 2017. Date of publication July 31, 2017. The associate editor coordinating the review of this manuscript and approving it for publication was Prof. Aleksandar Nešković.

This paper is a revised and expanded version of the paper presented at the 22nd Telecommunications Forum TELFOR 2014 [21].

This work was supported by Serbian Ministry of Science, Education and Technological Development under project TR32028 - "Advanced Techniques for Efficient Use of Spectrum in Wireless Systems"

Miloš Janjić, Student of PhD study, University of Belgrade - School of Electrical Engineering, milos.janjic@ic.etf.rs.

Miljko Erić, University of Belgrade - School of Electrical Engineering,meric@open.telekom.rs.
Orthogonal Frequency Division Multiplexing (OFDM) modulation technique is a strong candidate for implementation of a cognitive secondary link. Advantages of OFDM technology when employed by CR and challenges to a practical OFDM-based CR system are studied in [4].

Several techniques for the coexistence of OFDM and Frequency Hopping (FH) systems have been proposed in literature. The problem is mainly investigated in the context of coexistence of OFDM based WLAN (Wi-Fi) and Bluetooth using FH. One such technique is adaptive frequency hopping which is presently used by Bluetooth [5]. In this technique Bluetooth scans the channels, marks the bands occupied by interferer as "bad", and doesn't use them. Use of erasures [5]-[8] or deterministic nulling is a particularly valid technique for OFDM based systems to avoid narrowband interference in which the subcarriers significantly affected by the interference signal are replaced by null subcarriers, meaning nothing is transmitted on them. Another technique, [5], [9], consists of predicting the interference signal using null subcarriers and afterwards subtracting the estimated interference from each subcarrier. Adaptive change of the Wi-Fi packet length in the MAC layer was proposed in [10].

The vast majority of published papers related to the cognitive radio are theoretical. There are very few papers dealing with practical implementation. One such paper is [11], in [12]-[15] OFDM based Software Defined Radio (SDR) implementation is addressed, [16] contains the implementation of cognitive radio with $\mathrm{FH}$ primary users. The contribution of this paper is a practical implementation of FH and OFDM signals coexistence in the context of cognitive radio, using Universal Software Radio Peripheral (USRP) platforms. This means that first the secondary user (OFDM) has to obtain the primary user's (FH) parameters through spectrum sensing. Afterwards, the secondary user synchronizes to the primary user hopping, detects central frequency and bandwidth of each hop, adjusts its parameters and employs a technique similar to the above method of erasures to avoid making interference to the primary user, while ensuring good conditions for its own transmission. It is important to note that our primary user doesn't operate in a licensed part of the spectrum. Both the primary and secondary users operate in an unlicensed part of the spectrum, which corresponds to Bluetooth and Wi-Fi coexistence scenario, but, in order to have their operation coordinated, one system is designated primary (unaware of the other system presence), and the other secondary (has to adjust its transmission parameters). 
The rest of the paper is organized as follows. Section II presents our experimental infrastructure. Section III describes the implementation of OFDM and FH coexistence based on the principles of cognitive radio. Section IV contains the obtained experimental results. The future steps in the development of the secondary link are pointed out in Section V.

\section{DEVELOPMENT ENVIRONMENT}

This section discusses the hardware and software platform used for our solution. USRP (Ettus Research, USA) was used as SDR. USRP provides a digital baseband and IF section within the hardware, which aids utilizing general purpose computers to function as high bandwidth software radios. In addition, the board can provide an interface to various daughter-boards for a wide range of applications. The basic philosophy behind the USRP is to provide all waveform specific features like modulation and demodulation in CPU, whereas the high speed operations like interpolation, decimation, digital up and down conversion are provided within FPGA [11].

For communication with USRP, Matlab toolbox for USRP was used. Matlab toolbox communicates with USRP platform using UDP (User Datagram Protocol) protocol. Computer sends baseband signal samples to the USRP which generates a signal at a given frequency and bandwidth specified by interpolation factor. Signal is then transmitted over an antenna to the receiving end where all operations are reversely executed.

\section{IMPLEMENTATION}

In implementation, we started from an OFDM simulator presented in [17]. Operation of OFDM transmitter is described in detail in [18]. Before developing algorithm for the coexistence of secondary and primary signals, we implemented several algorithms to achieve transmission in real conditions: improved time synchronization, frequency offset estimation and compensation, channel estimation and equalization (secondary signal), estimation and correction of time-synchronization errors and channel equalization by using adaptive filter (primary signal). These algorithms are explained in [18].

\section{A. Primary signal - Frequency hopping}

Primary signal is generated in Matlab, saved in a file, which is used for simulation of primary signal at the location of secondary transmitter.

Primary user transmits a text message using Frequency Hopping (FH) and BFSK modulation. Guard intervals are inserted in between adjacent hops of primary signal, as in Bluetooth standard [6]. We assume that secondary signal bandwidth and overall primary signal bandwidth are equal. Primary signal uses a random number between 6 and 20 non-overlapping channels for frequency hopping with no frequency guard intervals. The bandwidth of every channel is equal (approximately overall bandwidth/number of channels) and defined by the first null criterion. Channels are equidistantly distributed in frequency spectrum. In order to reduce side lobes of the channels spectrum, prior to modulation pulses are shaped by a Gaussian filter with $B_{3} T=0.5$, where $B_{3}$ denotes Gaussian filter bandwidth and $T$ denotes a symbol length in samples. Spacing between frequencies of waveforms representing binary 0 and 1 is equal to $1 / T$. For the purpose of detecting the start of data stream by the Rx, in front of FH stream a header with a guard interval is inserted. The first half of the header is comprised of a complex sinusoid, later used for frequency offset estimation, whereas the second part are samples complying with Gaussian distribution used as a training sequence for adaptive filter. The adaptive filter with Least Mean Square (LMS) algorithm provides fine time synchronization, as well as channel equalization for primary signal. In order to enable adaptive filter proper operation, CFO compensation in training sequence is required. It is implemented as for OFDM signal using the sinusoid comprising the first part of the header. An optimum value of adaptive filter adaptation constant is achieved using a normalized LMS algorithm.

A quadrature receiver with matched filters is used for demodulation. Non coherent demodulation is used because ideal synchronization of the $\mathrm{Rx}$ to the incoming signal can't be provided because the response of the USRP after each guard interval is different and adaptive filter is used only at the beginning of the FH stream. In order to improve the probability of correct symbol detection, a moving average filter with length equal to half the symbol length was applied to envelope of the signals at the output of the matched filters.

\section{B. Coexistence of OFDM and FH systems}

OFDM and FH coexistence problem, with the respective spectrograms, is illustrated in Fig. 3. In our scenario, we have a secondary user, transmitting a 256-grayscale bitmap image, shown in Fig. 2, employing OFDM and 256 PSK modulation, and a primary user, transmitting a text message using $\mathrm{FH}$ and BFSK modulation.

The secondary Tx has no information about hop duration, or the number and bandwidth of channels used in the $\mathrm{FH}$ process of primary signal.

At a certain point in time the secondary $\mathrm{Tx}$ starts to perform spectrum sensing in order to obtain the above mentioned parameters of primary user transmission, which has started at some earlier point. The secondary Tx receives a sequence of the primary user signal of arbitrary length to be analyzed. For assessment of hop instant and duration, the algorithm with two sliding windows is used [18]. For estimation of the number and bandwidth of channels, an approach based on energy detection in signal spectrum is used. We assume that the secondary Tx and secondary Rx are in the same environment regarding the primary user signal. At some point the secondary Tx starts to send data. Because we don't have real primary user data, but it is generated in Matlab, transmit USRP emulates both, the primary $T x$ and secondary $T x$. It generates a file where the sum of the primary and secondary signals is saved, and the beginning of the secondary signal is shifted in time in accordance with the above. In this way the total signal at the secondary $\mathrm{Rx}$ is simulated. The length of one OFDM frame with guard interval is adjusted to match the length of one FH hop with its guard interval. Unlike the primary signal, where guard interval comes after useful data in a hop, in the secondary signal a guard interval precedes every data frame, and the start of OFDM guard interval is synchronized with the start of primary hop, as shown in Fig. 4. During OFDM guard interval the current FH channel 
is estimated. In the following frame the subcarriers occupying the bandwidth of the current primary signal hop (and a certain number of adjacent subcarriers) are not employed, as shown in Fig. 4.

In OFDM the $i$ th subcarrier signal is characterized by a power spectral density (PSD) of the form

$$
\Phi_{i}(f)=K \operatorname{sinc}^{2}\left(\left(f-f_{i}\right) T_{s}\right)
$$

where $K$ is a constant determined by the signal level, sinc is the sinc function defined as $\operatorname{sinc}(x)=\sin (\pi x) /(\pi x), f_{i}$ is the center frequency of the subcarrier, and $T_{s}$ is the OFDM symbol duration, which consists of the duration of one FFT block, $T$, and the guard interval, $T_{G}$. Assuming that the symbols in different subcarriers are independent of each other, the PSD of an OFDM signal is obtained as

$$
\Phi(f)=\sum_{i} \Phi_{i}(f)
$$

where the index $i$ includes all active subcarriers. Because of the relatively large side-lobes of the sinc pulse in Eq. 1, the out-of-band energy generated by an OFDM signal can be significant. This may result in unacceptable interference to primary users. The sinc shape of the subcarrier spectra is a consequence of the abrupt transition among successive OFDM symbols. One can avoid the sinc pulse by introducing soft transitions among successive symbols through cyclic extension of each OFDM symbol from $T_{s}$ to $(1+2 \beta) T_{s}$ [19], and windowing by a raised cosine shape of the form shown in Fig. 1., that is defined by [20]:

$$
g(t)= \begin{cases}\frac{1}{2}+\frac{1}{2} \cos \left(\pi+\frac{\pi t}{\beta T_{s}}\right), & \text { for } 0 \leq t<\beta T_{s} \\ 1, & \text { for } \beta T_{s} \leq t<T_{s} \\ \frac{1}{2}+\frac{1}{2} \cos \left(\frac{\pi\left(t-T_{s}\right)}{\beta T_{s}}\right), & \text { for } T_{s} \leq t<(1+\beta) T_{s},\end{cases}
$$

where $\beta$ denotes the roll-off factor.

The successive OFDM symbols are then overlapped as also shown in Fig. 1. As a result, the effective duration of each OFDM symbol is increased from $T_{s}$ to $(1+\beta) T_{s}$, which constitutes a bandwidth loss of $\beta /(1+\beta)$.

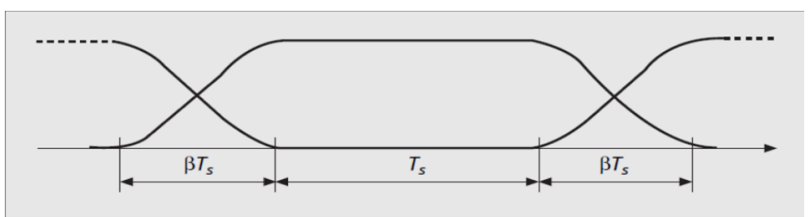

Fig. 1. OFDM pulse shaping window [19].

Even though the raised cosine window is very effective in reducing the side-lobes of the subcarrier spectra, the sidelobes that are close to the main lobe are still large. Hence, in addition to the subcarriers that fall in the current primary channel band, a number of subcarriers in vicinity of primary channel band are deactivated as well.
These two measures to protect a primary user from a secondary user can produce benefits in other direction, i.e. to a secondary user, because we can raise the level of secondary signal without making more interference to a primary user relative to the case without pulse shaping and neighboring carriers deactivation. Of course, these measures cause some spectrum efficiency loss, so a rational compromise is needed.

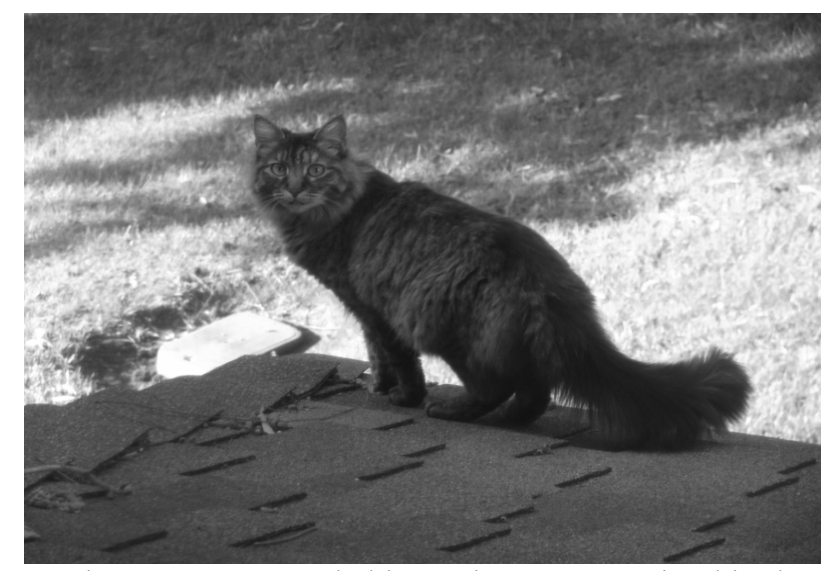

Fig. 2. 256-grayscale bitmap image transmitted in the experiments.

At the receiver side spectrum sensing with real signals is performed (detection which channel occupies the current hop), as well as demodulation and data display of both primary and secondary signal. The receiver synchronizes to FH signal using the algorithm with two sliding windows [18]. The header preceding FH signal is used for this purpose. The adaptive filter provides fine time synchronization. At the start of every hop, Rx checks for the presence of OFDM header, placed in front of OFDM data stream. Based on the channel used in the current hop, the secondary Rx knows which of the two predefined sinusoid frequencies the secondary Tx has used in the header. We use two different frequencies to avoid a situation where the frequency of the sinusoid is inside the current hop bandwidth of the primary signal. Before every OFDM frame demodulation, estimation and correction of CFO is done, because experiments have shown that $\mathrm{CFO}$ can be unstable during transmission. For this purpose a piece of header is sent in front of every OFDM frame (red spots in Fig. 3). As in the case of the header in front of OFDM data stream, coinciding of sinusoid frequency with the current primary channel is avoided at the Tx. During guard intervals preceding every OFDM frame the receiver performs spectrum sensing by estimating the channel of the current hop. It is assumed that the receiver has all the information the Tx has obtained by spectrum sensing, as well as the decisions the Tx has made afterwards (i.e., the exact number of useful subcarriers in one frame), so that the $\mathrm{Rx}$ is able to get information concerning the subcarriers used for data transmission at the Tx in the current frame. If compared to spectrum sensing on the Tx side, spectrum sensing on the Rx side has less work to do, but it is carried out on real signals. 


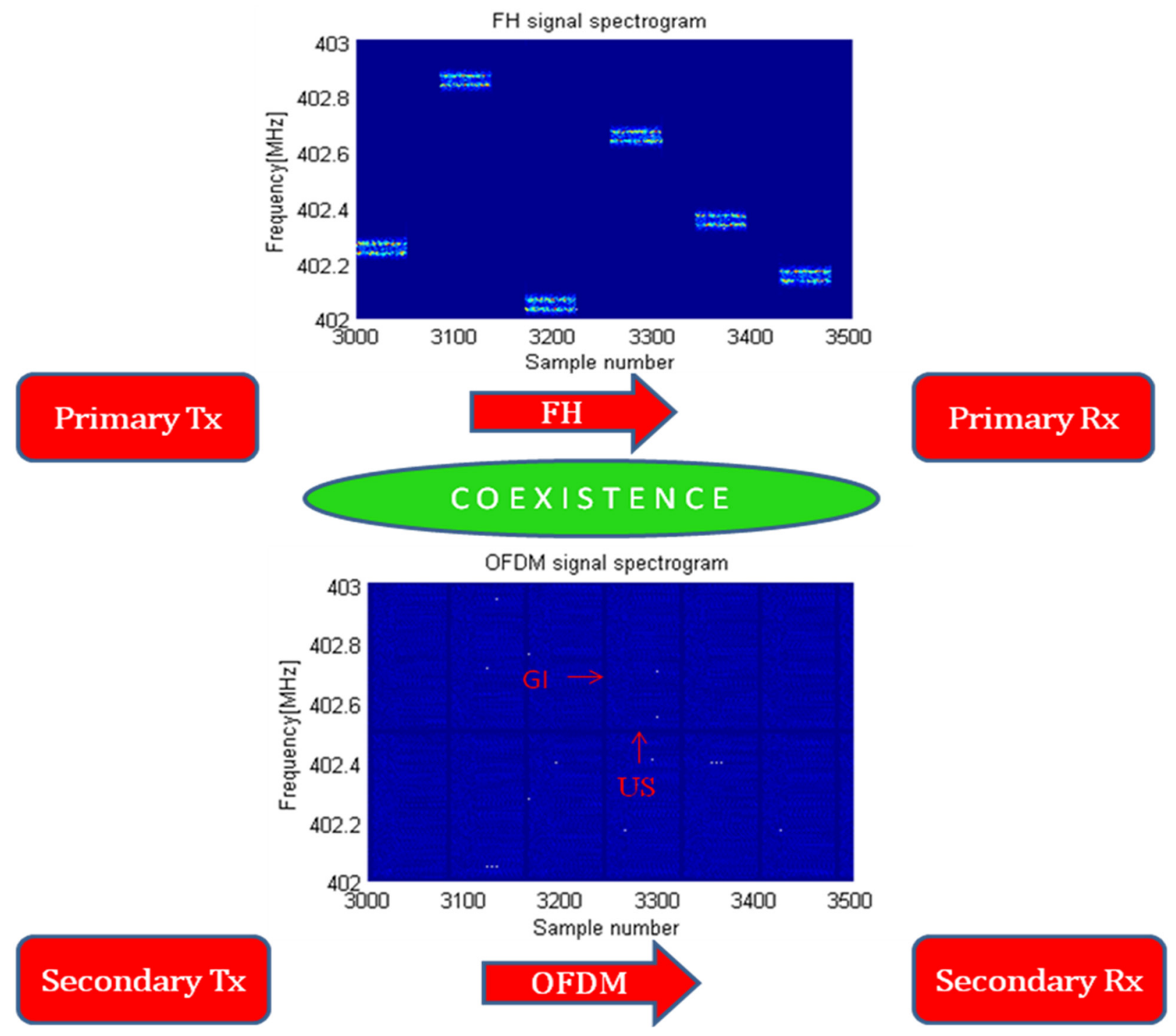

Fig. 3. FH and OFDM signals coexistence problem. FH signal is sparsely distributed over the frequency-time plane (the rectangles in the spectrogram). OFDM signal fills all the frequency-time plane except for unused subcarriers around the DC component (US) and time guard intervals between the frames (GI).

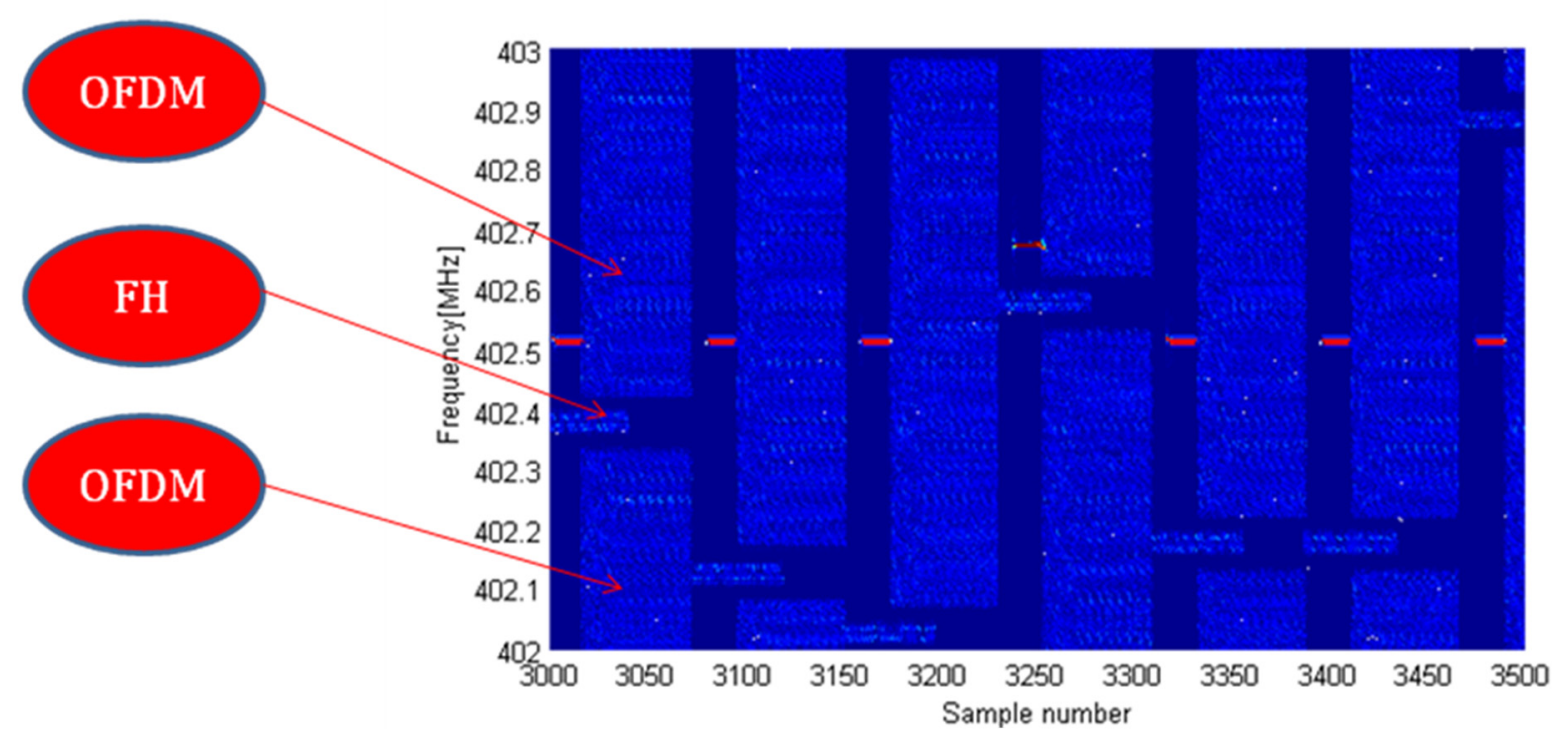

Fig. 4. A solution to the coexistence problem of OFDM and FH signals: spectrogram of the signal portion at the Rx containing several OFDM frames/FH hops. 


\section{EXPERIMENTAL RESULTS}

This section presents the results obtained by experiments conducted in a laboratory environment, deploying 2 laptops controlling the transmit and receive N210 USRP platforms, respectively, as shown in Fig. 5.

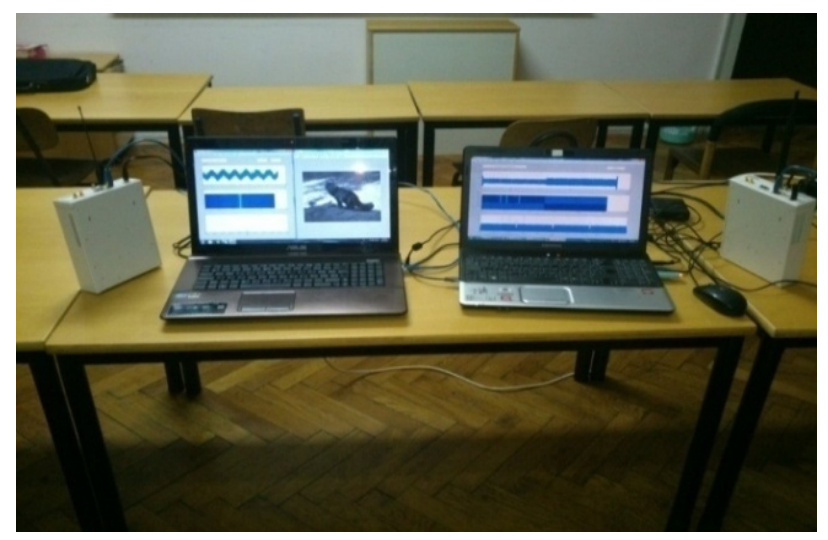

Fig. 5. Development environment.

Transmission parameters for the secondary user were: 402.5 MHz carrier frequency, bandwidth of $1 \mathrm{MHz}$, OFDM with 1024 subcarriers, 256 PSK modulation on subcarriers. Transmission parameters for the primary user were: 402.5 $\mathrm{MHz}$ carrier frequency, overall bandwidth of $1 \mathrm{MHz}$, BFSK modulation with slow frequency hopping and 20 nonoverlapping channels. Fig. 6 shows the real part of time domain baseband samples, spectrogram and frequency spectrum of the RF signal at the transmitter, respectively. Fig. 9 gives the corresponding displays for the signal at the receiver, as well as obtained image and text data at the receiver.

OFDM and FH mean power ratio was $12 \mathrm{~dB}$, where for OFDM power on bandwidth equal to instantaneous bandwidth of primary signal is considered. Roll-off factor for OFDM symbols was set to 0.05 , whereas the number of deactivated subcarriers expressed in primary channel bandwidth was 2.6. At the output of Tx USRP signal was scaled to avoid amplitude clipping. USRP transmitted data blocks of the length equal to one hop with guard interval. Rx USRP received data blocks of the same size. We used Local Oscillator (LO) offset value of $10 \mathrm{MHz}$ in Tx and $\mathrm{Rx}$ USRP to move the signal bandwidth away from interference generated by the USRP hardware.

Percent of incorrectly detected symbols for the secondary user was 3.2, whereas the primary user signal was demodulated without errors.

Fig. 7 gives the normalized estimated channel transfer function at one of the OFDM frames. It is noticeable that subcarriers around central frequency, and at the edges of the frequency band were not used, as well as subcarriers corresponding to frequencies occupied by the primary user.

Fig. 8 shows estimated channel transfer function, transfer function of the adaptive filter, as well as, overall transfer function, obtained by multiplying these two transfer functions, used for fine time synchronization and channel equalization in the transmission of the primary user signal.

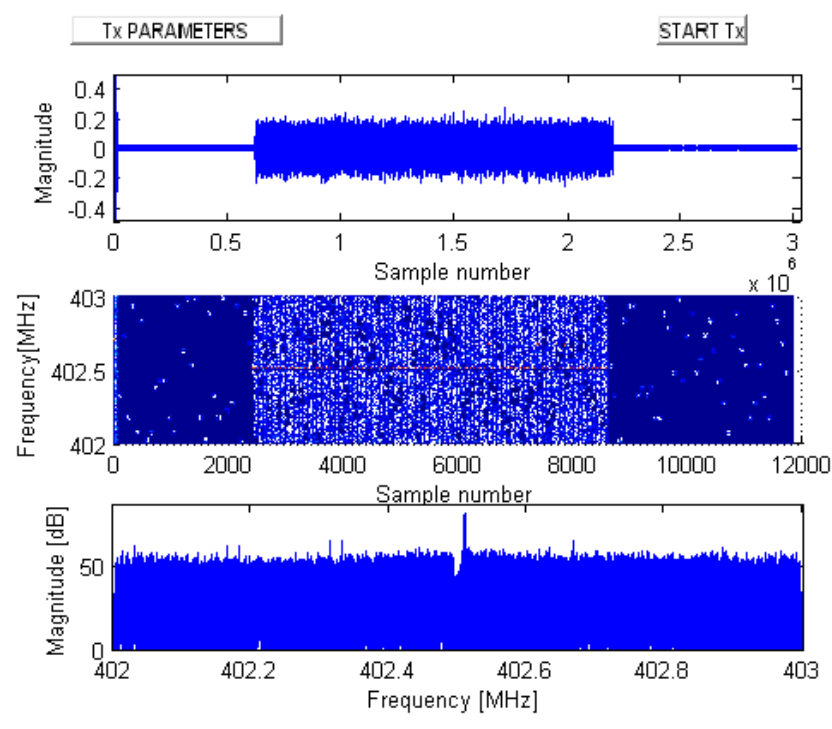

Fig. 6. Time domain samples, spectrogram, and spectrum of the transmitted signal.

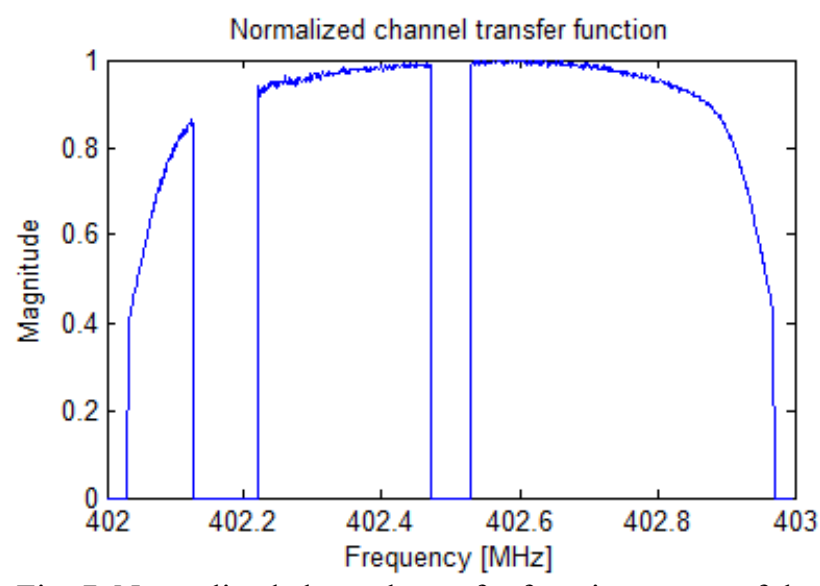

Fig. 7. Normalized channel transfer function at one of the OFDM frames.

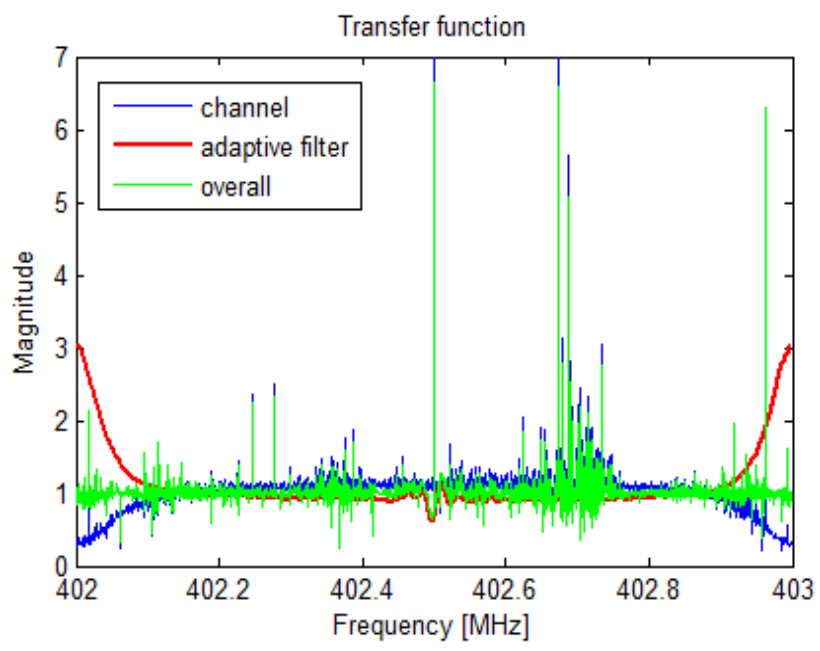

Fig. 8. Channel transfer function, adaptive filter transfer function, and overall transfer function. 


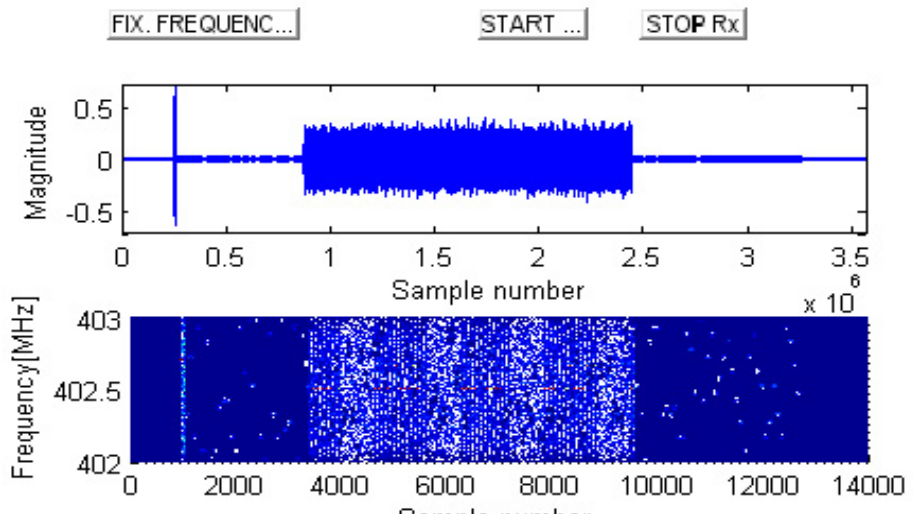

\section{Secondary User - OFDM}
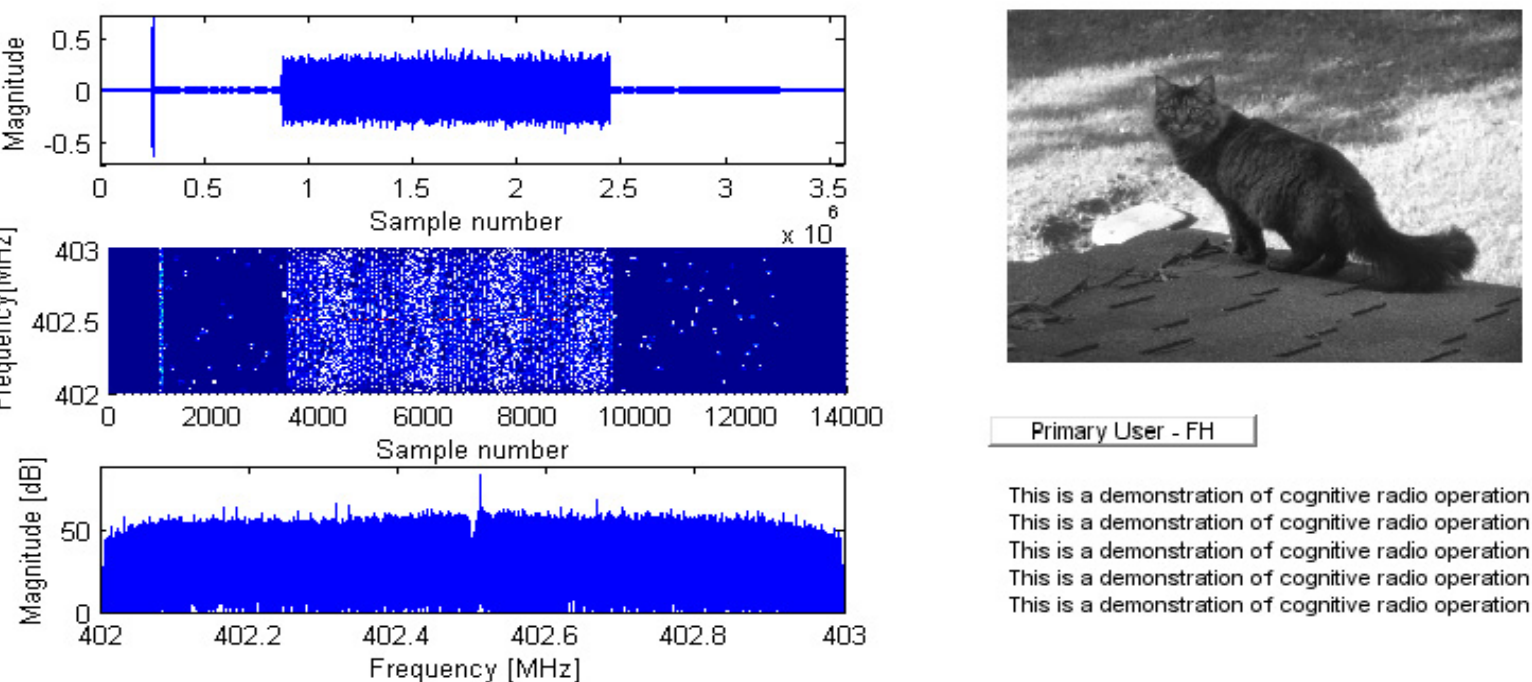

\section{Primary User - FH}

This is a demonstration of cognitive radio operation. This is a demonstration of cognitive radio operation. This is a demonstration of cognitive radio operation. This is a demonstration of cognitive radio operation. This is a demonstration of cognitive radio operation.

Fig. 9. Time domain samples, spectrogram, spectrum of the received signal, demodulated image and text data in the receiver after transmission using 256 DPSK OFDM and BFSK FH, respectively.

\section{CONCLUSIONS AND FUTURE WORK}

In this paper our technical solution for a secondary link has been presented. We have implemented the coexistence of secondary user (OFDM) and primary user (FH) in real indoor conditions. We have realized cognitive features of spectrum sensing and changing transmission parameters. But there are still problems to be solved, which will be our future work. The next steps in our project are: further increase in the speed of operation of the transmitter and receiver, implementation of complete spectrum sensing at the $\mathrm{Rx}$, further automation of the Tx and $\mathrm{Rx}$, solving a problem of buffering samples from USRP platform, a greater level of controllability of our application. Implementation of the whole mechanism in FPGA technology using ML605 platform and NUTAQ modules serving as analog front-end would be final steps to maximize the speed of the application and thus enable its use in real-life conditions.

\section{REFERENCES}

[1] F. F. Digham, "Joint power and channel allocation for cognitive radios," in Proc. Wireless Comm. Networking Conference, pp. 882887, Las Vegas, NV, USA, Apr., 2008.

[2] J. Mitola, "Cognitive radio: an integrated agent architecture for software defined radio," PhD thesis, Royal Institute of Technology (KTH), Stockholm, Sweden, 2000.

[3] T. Yücek and H. Arslan, "A survey of spectrum sensing algorithms for cognitive radio applications," IEEE Comm. Surveys and Tutorials, vol. 11, no. 1, pp. 116-130, 2009.

[4] H. Mahmoud, T. Yücek, and H. Arslan, "OFDM for cognitive radio: Merits and challenges," IEEE Wireless Comm., vol. 16, no. 2, pp. 614, Apr. 2009

[5] M. Roy, "WLAN interference cancellation from OFDM based WLAN: Strategic comparisons of coexistence techniques," Cyber Journals, JSAT, Sept. 2011

[6] K. K. Wong and T. O'Farrell, "Coexistence of 802.11 WLANs with bluetooth," University of Leeds, UK

[7] A. Amurugam, A. Doufexi, A. Nix, and P. Fletcher, "An investigation of the coexistence of $802.11 \mathrm{~g}$ WLAN and high data rate bluetooth enabled consumer electronic devices in indoor home and office environments," IEEE Trans. on Consumer Electronics, vol. 49, pp. 587-596, Aug. 2003.
[8] S. Abukharis, J. A. Alzubi, O. A. Alzubi, and S. Alamri, "Packet error rate performance of IEEE 802.11g under Bluetooth interface," Research Journal on Applied Science, Engineering, and Technology, vol. 8 (12), pp. 1419-1423, 2014.

[9] D. Zhang, P. Fan, and Z. Cao, "Interference cancelation for OFDM systems in presence of overlapped narrowband transmission System," IEEE Trans. on Consumer Electronics, vol. 50, no. 1, pp. 108-113, Feb. 2004

[10] A. C. C. Hsu, D. S. L. Wei, C. C. J. "Coexistence Wi-Fi MAC design for mitigating interference caused by collocated bluetooth," IEEE Trans. on Computers, vol. 64 (2), pp. 342-352, Feb. 2015.

[11] V. V. Patcha, "Experimental study of cognitive radio test-bed using USRP", thesis by B. TECH in Electronics and Communication Engineering Padmasri Institute of Technology, India, Aug. 2011.

[12] A. Marwanto, M. A. Sarijari, N. Fisal, S. K. S. Yusof, and R. Rashid, "Experimental study of OFDM implementation utilizing GNU radio and USRP - SDR," Proc. of the 9th IEEE Malaysia Inter. Conf. on Comm., Kuala Lumpur, Malaysia, Dec., 2009.

[13] I. Singh, "Software defined radio implementation of improved OFDM system," Master thesis, Thapar University, Patiala, Punjab, India, Aug. 2013.

[14] D. T. Nguyen, "Implementation of OFDM systems using GNU Radio and USRP," Master thesis, University of Wollongong, Australia, July 2015.

[15] T. Magounaki, "A software-defined implementation of an OFDMadaptive OFDMA system using USRPs 1," Master thesis, School of Electronic and Computer Engineering, Technical University of Crete, Greece, Oct. 2014

[16] A. Jain, V. Sharma, and B. Amrutur, "Soft real time implementation of a cognitive radio testbed for frequency hopping primary satisfying QoS requiremets," Twentieth National Conf. on Comm., NCC 2014, Kanpur, India, Feb. 2014.

[17] P. G. Lin, "OFDM simulation in MATLAB", California Politechnic State University, San Luis Obispo, June 2010.

[18] M. Janjić, M. Brkovic, and M. Erić, "Development of OFDM based secondary link: Some experimental results on USRP N210 platform”, Telfor Journal, vol. 6, no.1, pp.30-35, 2014.

[19] B. Farhang-Boroujeny and R. Kempter, "Multicarrier communication techniques for spectrum sensing and communication in cognitive radios", Commun. Mag., vol. 46, no. 4, pp. 80-85, 2008.

[20] T. Weiss, J. Hillenbrand, A. Krohn, and F. Jondral, "Mutual interference in OFDM-based spectrum pooling systems", in Vehicular Technology Conference Spring, vol. 4, 2004, pp. 18731877, Milan, Italy, May 2004.

[21] M. Erić and M. Janjić, "A solution to coexistence of OFDM and FH signals based on principles of cognitive radio implemented on USRP N210 platform," 2014 22nd Telecommunications Forum Telfor (TELFOR), Belgrade, 2014, pp. 276-279. 